\title{
EL RECONOCIMIENTO HIDROLÓGICO DEL VALLE DEL EBRO EFECTUADO POR PEDRO ANTONIO DE MESA EN 1863*
}

\author{
R. Galván Plaza \\ Confederación Hidrográfica del Ebro (Ministerio de Medio Ambiente) \\ P. Sagasta, 24-26, 50071 Zaragoza \\ rgalvan@chebro.es
}

\begin{abstract}
Resumen: La memoria del "Reconocimiento del valle del Ebro" elaborada por Pedro Antonio de Mesa en 1865 como colofón de los trabajos de las brigadas hidrológicas de la Junta General de Estadística, constituye un documento inapreciable por la descripción geográfica, principalmente hidrológica pero no sólo, que realiza de la cuenca del Ebro. Un análisis en detalle del mismo permite la valoración adecuada de la labor pionera de Antonio de Mesa, así como servir de testimonio de referencia de la evolución operada en la cuenca del Ebro desde entonces.
\end{abstract}

\begin{abstract}
The report untitled "Reconocimiento del valle del Ebro" made by Pedro Antonio de Mesa in 1865 as a final step of the works carried out by the hydrological brigades of the Junta General de Estadística, is an outstanding document because of the geographical description of the Ebro basin at that time, not only hidrologicaly speaking but in a general perspective. A detailed analysis of this document allows us to make a proper evaluation of the pioneering work of Antonio de Mesa and consider it as a reference of evolution that has taken place in the Ebro basin since then.
\end{abstract}

Palabras clave: cuenca del Ebro, Antonio de Mesa, aforos, evolución del regadío.

\section{Introducción y antecedentes}

Según iba consolidándose el régimen liberal en España, se aprecia la necesidad de poner en producción todos los recursos del país para recuperar su maltrecha eco-

* Recibido: 13-02-08. Aceptado: 17-06-08. 
nomía, por lo que el conocimiento e inventario de su territorio y recursos naturales se convierte en uno de los objetivos de la administración de la época. La ley de Medición del Territorio de 1859, aprobada durante el "gobierno largo" de O'Donnell, pone en manos de la Junta General de Estadística, creada en 1856, la ejecución de trabajos geográficos de reconocimiento del medio físico, entre los que se encuentra la riqueza hidrológica. Con este motivo se ponen en marcha las brigadas hidrológicas con ambiciosos objetivos, entre los que estaban la elaboración de cartografías, aforos y descripción de ríos y sus aprovechamientos. Siendo los medios para la consecución de dichos objetivos enormemente limitados, éstos sólo se alcanzaron muy parcialmente.

Por ello, para evitar que tales esfuerzos se diluyeran en el intento de abarcar demasiado, el ingeniero-jefe de las operaciones hidrológicas de la Junta General de Estadística entre 1862 y 1865, Pedro Antonio de Mesa, propuso reducir las operaciones inicialmente previstas a simples reconocimientos de los grandes valles y a concentrar las operaciones en la realización de aforos, midiendo los caudales de estiaje, a los que se consideraba claves para el posible aprovechamiento de las aguas.

Fruto de sus ideas se llevaron a cabo reconocimientos hidrológicos del Duero, Tajo, Guadalquivir y Ebro, llegándose a publicar sendos informes de los dos últimos. Se trataba de un trabajo "completamente nuevo en España (y no muy adelantado fuera de ella)" (Antonio de Mesa, 1864), sólo precedido por los trabajos parciales de las Comisiones de Estudio del Ministerio de Fomento.

El reconocimiento hidrológico del Valle del Ebro tuvo lugar en el verano de 1863, iniciándose el 4 de julio de 1863 y finalizándose posiblemente en octubre, sin que quede constancia de la fecha precisa. El informe de dicho reconocimiento fue publicado en 1865.

Se trataba de una labor casi de explorador y no cabe duda del tremendo esfuerzo que a lomos de caballería significó el reconocimiento, no sólo por el número de kilómetros, que no debió de bajar de 4.000 o 5.000, habida cuenta de los puntos de aforo y de lo que él mismo referiría para el caso del Guadalquivir acerca de la necesidad de cubrir $40 \mathrm{~km}$ diarios o de sus propias afirmaciones sobre como entre 1862 y 1865 recorrió unos $20.000 \mathrm{~km}$ a caballo por las márgenes de los ríos Guadalquivir, Ebro, Duero y Guadiana (Mateu Bellés, 1996). A ello se añade la dificultad de las comunicaciones de la época y la necesidad de seguir los cursos de los ríos. Dificultades que describe en ocasiones, como cuando menciona el paso por el estrecho de Besantes entre Álava y Burgos, "siguiendo el curso del río por la peligrosa senda que los Carabineros tienen establecida para evitarse el gran rodeo del camino ordinario" (Antonio de Mesa, 1865). Del carácter poco más que exploratorio también encontramos ejemplos, como cuando no logra aforar el Aragón en su origen "por los malos 
informes de un guí, que nos dio a conocer este río en uno de sus afluentes, de cuyo error me apercibi cuando no era fácil remediarlo" (op.cit).

\section{El reconocimiento del valle del Ebro}

El contenido de la memoria del "Reconocimiento del Valle del Ebro" ha sido sintetizado y valorado por Joan F. Mateu en su minucioso y brillante trabajo "Las brigadas hidrológicas de la Junta General de Estadística (1859-1867)" (Mateu Bellés, 1996) que además es básico para conocer la génesis y toda la labor hidrológica de la Junta General de Estadística.

Las primeras páginas del documento de Antonio de Mesa, se dedican a la descripción física de la cuenca: dimensiones, pendiente general, clasificación hidrológica de afluentes, caracterización geológica; complementado con datos de población y superficie cultivada.

La parte central de la descripción presenta los resultados de los aforos practicados en el estiaje de 1863 bajo el concepto de que dicho estiaje sería equivalente a la riqueza hidrológica que todavía podía ser aprovechada. Describe con precisión cada aforo practicado y los contrasta entre sí para obtener finalmente lo que, en su propia terminología, resulta ser la producción total del valle del Ebro que cifra en $274,2 \mathrm{~m}^{3} / \mathrm{s}$, de la cual $125 \mathrm{~m}^{3} / \mathrm{s}$ se pierden al mar y no es aprovechada. Incorpora además otros datos y noticias de aprovechamientos y concesiones, usando la documentación existente en la Junta de Estadística y en la Dirección General de Obras Públicas. Combina el trabajo de campo con el de gabinete para dar una mejor radiografía del valle. No olvida tampoco dar noticia de las aguas estancadas, minerales y y salinas; y dedica también un espacio a las comunicaciones existentes y previstas. Este núcleo central del informe lo subdivide en tres partes o regiones: Superior, hasta Miranda de Ebro, Media, de Miranda a Zaragoza e Inferior, de Zaragoza a la desembocadura.

Por el interés que pudiera tener, transcribe la memoria del ingeniero francés Lesguiller sobre los trabajos para la navegación del Ebro, a la que critica abiertamente por su falta de realismo, y finaliza con unas conclusiones como resultado de todo lo expuesto en las que resume todas las características descriptivas del valle del Ebro, ya recopiladas de la documentación existente, ya fruto del propio reconocimiento: superficie, población, longitud de ríos, caudales, superficie regada y la susceptible de ser promovida, artefactos fabriles, baños, extracciones de sal y vías de comunicación.

$\mathrm{Al}$ informe se acompañan dos mapas: un "Perfil general del Ebro y de sus principales afluentes" y un "Plano general del Ebro y sus afluentes" a escala 1:1.000.000 
(Fig. 1), seguramente el primero en que se representa la cuenca del Ebro de tal modo, dibujado a partir de la cartografía que en fechas recientes había elaborado Francisco Coello.

\section{La hidrología y los aforos practicados}

En el documento se recogen datos de 231 aforos en ríos, y alguno más en acequias y canales. De 129 de ellos, que como tales vienen enumerados en el informe, se muestra mayor detalle con el dibujo de la sección, recogiendo datos de ancho, superficie mojada y velocidad.

En el Plano general de la cuenca, ya citado, que acompaña al informe, vienen situados estos 129 puntos aforados considerados sin duda como de mayor importancia por el autor. En este trabajo se han dado coordenadas aproximadas a estos y a los

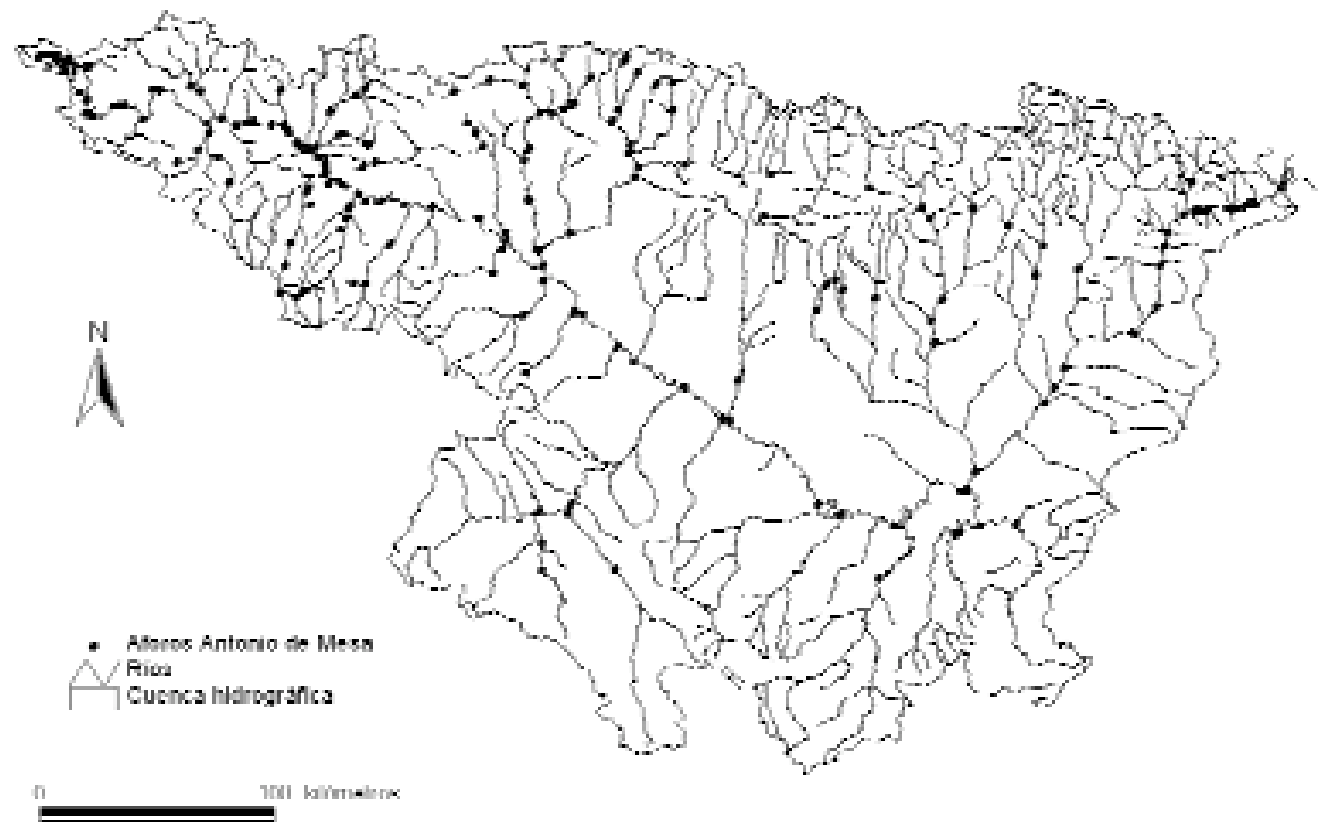

Figura 1. Plano general del Ebro y sus afluentes. Reconocimiento hidrológico del valle del Ebro. Pedro Antonio de Mesa, 1865.

Fuente: Obra citada. 
otros obteniendo como resultado una imagen final de todos los puntos aforados durante el reconocimiento (Fig 2). Se aprecia una mayor densidad de puntos en la cabecera, seguramente por no ser plenamente consciente del tiempo que había de llevarles recorrer toda la cuenca al iniciar las operaciones, y también por la margen izquierda, que alberga los afluentes más caudalosos. De hecho se presta menos atención a los afluentes de la margen derecha, puesto que como en el caso del Cidacos "no teniendo influencia en el régimen general del valle... ... no se recorrió su cauce... ...necesitando el tiempo para otros de mayor importancia" (Antonio de Mesa, 1865), y en definitiva porque el "Huerva, el Aguas (Vivas), Rí-Martín, Guadalope y Matarayas (Matarraña), son insignificantes al lado de aquellos (los de la margen izquierda), y la poco agua que producen en verano se consume y no alcanza a cubrir las necesidades ya creadas" (op.cit).

En la memoria del Reconocimiento Hidrológico del Valle del Guadalquivir, Antonio de Mesa explica el procedimiento de aforo, y que no repite, por no reiterarse, en la memoria del Valle del Ebro, dejando claro que "se ban becho de la manera más rápida y expedita" (Antonio de Mesa, 1864). Con todo, se detecta un extremado cuidado en la práctica y en las comprobaciones y búsqueda de concordancia de resultados, no aforándose cuando las circunstancias no son propicias, como cuando en el Ega "no habiendo encontrado sitio a propósito para hacerlo y no teniendo tiempo que perder en buscarlo, tuve que abandonar esta cuestión" (Antonio de Mesa, 1865) o cuando en el eje del Ebro explica que la concordancia entre los aforos efectuados aguas arriba y aguas abajo "no se ha obtenido de primera vez, pues ha babido con frecuencia que repetir muchos aforos y otros escluirlos (sic) del cuadro que presentamos" (op.cit).

No cabe duda de que el propio Antonio de Mesa, y otros ingenieros contemporáneos suyos, se daban cuenta de las limitaciones de las mediciones esporádicas y de las comisiones temporales para lograr la descripción certera del régimen de un río, así como de la imprecisión que podrían tener los aforos efectuados. Se trata de una duda que reflejaron estudios posteriores de los que es buen ejemplo el proyecto del Pantano del Ebro, en el valle de Campoo en Cantabria, elaborado por Manuel Lorenzo Pardo en 1916, que fuera luego el primer director de la Confederación Hidrográfica del Ebro, en el cual se refiere la mejora habida los procedimientos de aforo desde entonces y se analizan los datos aportados por Antonio de Mesa en la cabecera del Ebro indicando que "las cifras consignadas en el notable estudio citado eran notoriamente exageradas como expresión de los correspondientes caudales minimos de estiaje" (Lorenzo Pardo, 1916), y también que, en conjunto, "la relación media entre los caudales consignados en el libro del Sr. Mesa y los minimos reales de estiaje oscila alrededor del valor 2, el cual es superado en muchos arroyos y riachuelos, y en la parte menos caudalosa del río principal, y de sus afluentes más importantes" (op.cit). 


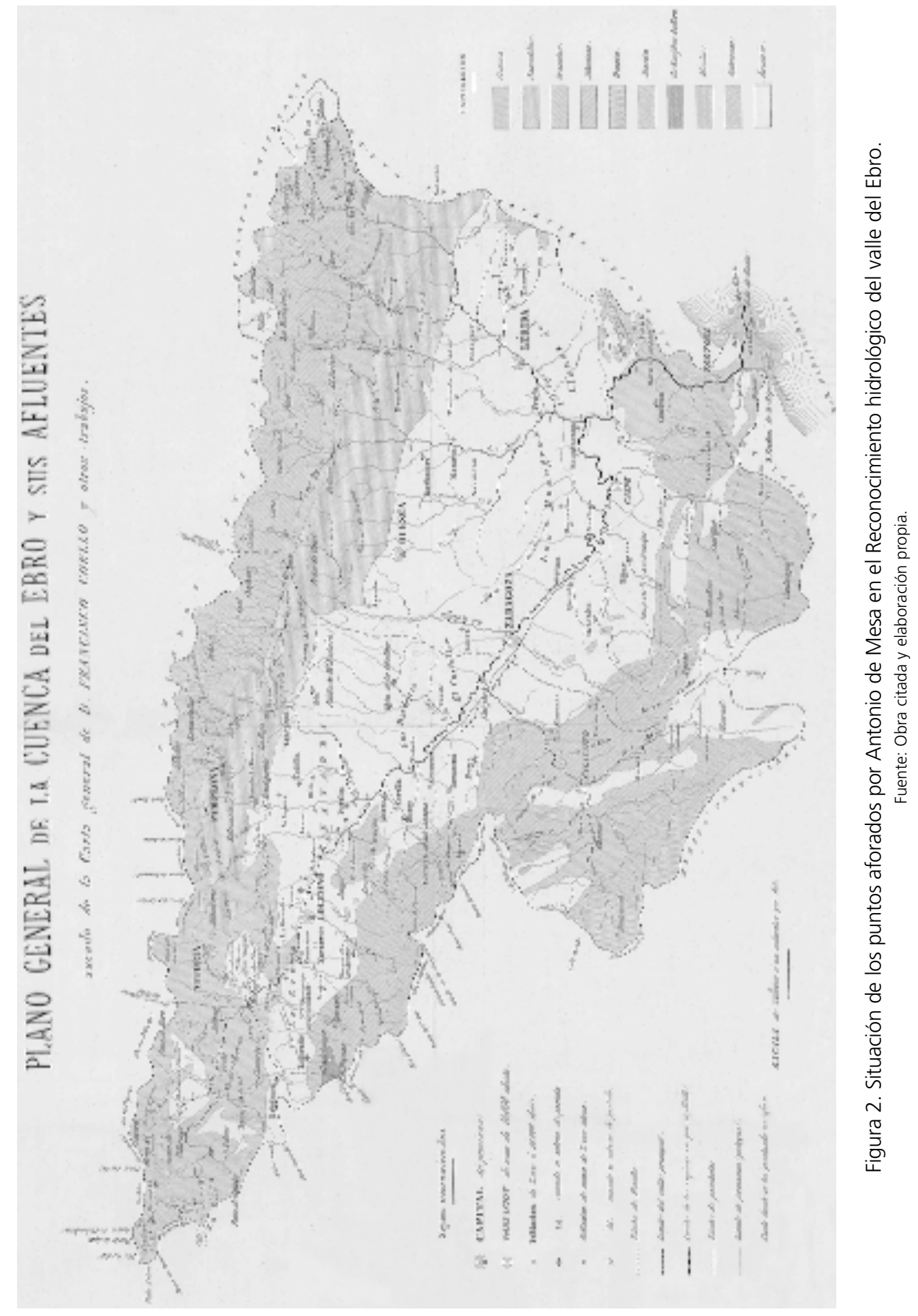


La duda sobre la exactitud de los aforos practicados por Antonio de Mesa no puede soslayarse. Sin embargo, al realizar una confrontación de los datos que obtuvo con los caudales registrados en la Red Oficial de Estaciones de Aforo para el periodo 1912-1947, antes de la construcción del embalse del Ebro y en el que las afecciones por consumos de agua en los regadíos todavía no excedían grandemente a aquellas del XIX, muestran que los datos de Mesa, dentro de su limitación, no eran ni mucho menos descabellados y se encuentran dentro de un orden de magnitud razonable (Tablas 1 y 2 ).

Los trabajos comenzaron el 4 de julio de 1863 en el arroyo Trisuerra en Cantabria, en los torrentes que dan nacimiento al río Híjar y por tanto al Ebro. La fecha de finalización no se recoge y tan sólo la referencia a los últimos aforos que se hicieron "estando ya la estación muy adelantada" (Antonio de Mesa, 1865), nos llevan a comienzo del otoño, seguramente octubre, al igual que en el caso del Guadalquivir, lo cual es más que plausible dado que el 21 de julio se encontraban todavía en Miranda de Ebro, la otra única fecha que figura en el documento.

Es reseñable cómo los riegos ya entonces afectaban fuertemente al caudal de estiaje y en numerosos puntos, principalmente de la margen derecha, llegaban a dejar completamente seco el río. Se trata por ejemplo del caso del río Iregua en su desembocadura que "estaba totalmente seco, no porque no produzcan aguas sus vertientes, sino porque en su totalidad se consumen en los riegos establecidos en su parte superior" (Antonio de Mesa, 1865). Es también el caso del Cidacos "que en la época de nuestro reconocimiento encontramos completamente seco en Calaborra y Autol' (op.cit). Lo mismo sucede con el otro Zidacos, el afluente del Aragón, "que ya se encontró seco en Tafalla" (op.cit). También es el caso del Alhama en el cual "cualquiera que sea la cantidad de aguas de este río en estiage (sic), toda se consume en los riegos establecidos, pues a su paso por Alfaro lo encontramos seco" (op.cit), e idéntico caso es el del Queiles que "llega al Ebro en Tudela sin una gota de agua" (op.cit). Otro caso es el del Arba "por el cual solo corrían en su desembocadura las aguas producidas por las filtraciones del Canal de Tauste" (op.cit). En el Gállego en desembocadura aunque se miden $9 \mathrm{~m}^{3} / \mathrm{s}$ también se dice que, "según los informes tomados, llega a secarse totalmente algunos veranos, lo cual no parece probable" (op.cit), informes que como se ve se consideran dudosos para este caso, pero que dan que pensar.

En la tabla 3, se recogen todos los resultados de los aforos efectuados. Como resumen final, en el Ebro en Flix se aforan 136,017 $\mathrm{m}^{3} / \mathrm{s}$, y aforando también el Canal de la Margen Derecha del Delta que parte de Cherta obtiene 11,552 $\mathrm{m}^{3} / \mathrm{s}$, lo que le permite afirmar que el caudal en desembocadura, "el agua que se perdía en el mar" (op.cit), era de $125 \mathrm{~m}^{3} / \mathrm{s}$, si bien en cuanto se completaran los riegos del Canal de Urgel, que habían comenzado su funcionamiento un año antes en 1862, indica que quedarían reducidos a $100 \mathrm{~m}^{3} / \mathrm{s}$. En resumen, sus mediciones le llevarán a afirmar que 


\begin{tabular}{|c|c|c|c|c|c|c|c|c|c|c|c|c|}
\hline & : & 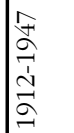 & 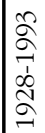 & 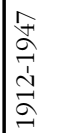 & 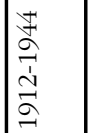 & 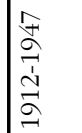 & 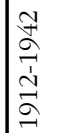 & 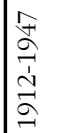 & 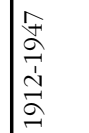 & 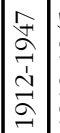 & 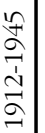 & 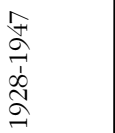 \\
\hline & 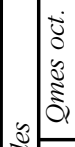 & 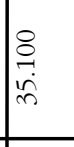 & 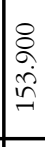 & 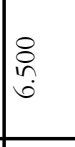 & 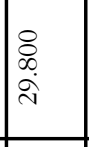 & 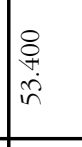 & 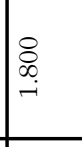 & $\begin{array}{l}8 \\
0 \\
\text { in } \\
\text { in }\end{array}$ & $\begin{array}{l}8 \\
1 \\
\infty \\
\infty\end{array}$ & 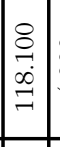 & 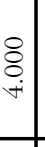 & 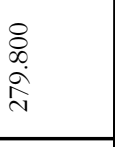 \\
\hline & 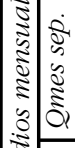 & \begin{tabular}{l}
8 \\
0 \\
0 \\
$\infty$ \\
0 \\
\hdashline
\end{tabular} & \begin{tabular}{|l}
0 \\
$\vdots$ \\
$\infty$ \\
0 \\
0
\end{tabular} & $\underset{+}{\stackrel{一}{+}}$ & 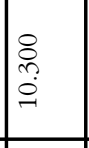 & 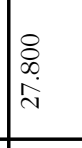 & $\underset{-i}{\stackrel{8}{0}}$ & 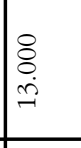 & 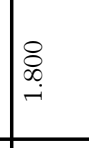 & 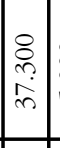 & & בְ. \\
\hline & 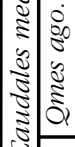 & 足 & \begin{tabular}{l}
8 \\
0 \\
\multirow{6}{0}{}
\end{tabular} & $\begin{array}{l}8 \\
\infty \\
\infty \\
\end{array}$ & 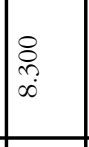 & 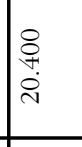 & : & 总 & 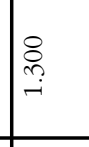 & $\mid \begin{array}{l}\stackrel{8}{0} \\
0 \\
\stackrel{4}{*}\end{array}$ & 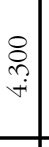 & 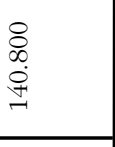 \\
\hline & 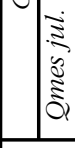 & 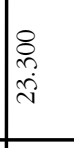 & 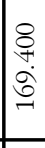 & $\begin{array}{l}\& \\
\stackrel{2}{1} \\
\text { in }\end{array}$ & \begin{tabular}{|}
$\stackrel{8}{8}$ \\
$\stackrel{\leftrightarrow}{+}$ \\
-
\end{tabular} & $\mid \begin{array}{l}\stackrel{8}{1} \\
\stackrel{\sim}{n}\end{array}$ & $\mid \begin{array}{c}\mathscr{Q} \\
\infty \\
-i\end{array}$ & $\mid \begin{array}{l}8 \\
\stackrel{+}{0} \\
\stackrel{0}{0}\end{array}$ & 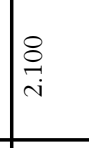 & 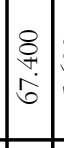 & 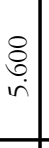 & 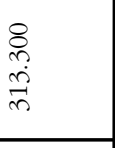 \\
\hline 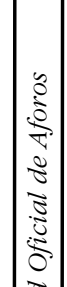 & 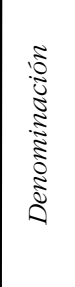 & 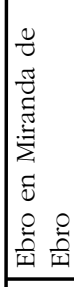 & 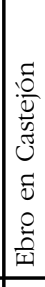 & 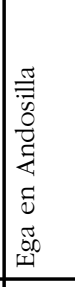 & 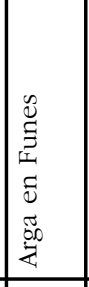 & 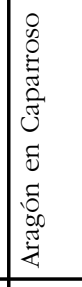 & 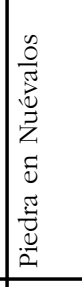 & 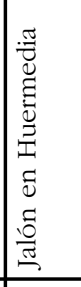 & 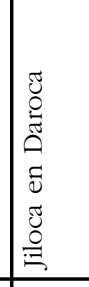 & 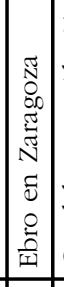 & 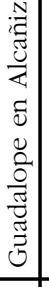 & 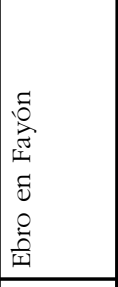 \\
\hline$\approx$ & 品 & - & v & $n$ & $\psi$ & in & $\infty$ & $a$ & 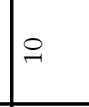 & $\Rightarrow$ & $\approx$ & 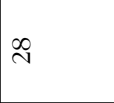 \\
\hline & 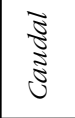 & 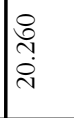 & 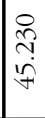 & $\mid \begin{array}{l}\stackrel{\overbrace{}}{.} \\
\text { ì }\end{array}$ & 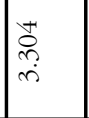 & 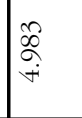 & 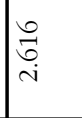 & $\mid \begin{array}{l}\stackrel{9}{1} \\
\infty \\
\infty\end{array}$ & $\begin{array}{l}2 \\
\text { in } \\
\text { n. }\end{array}$ & 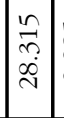 & 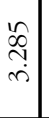 & 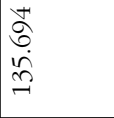 \\
\hline 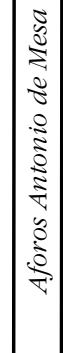 & 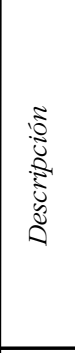 & 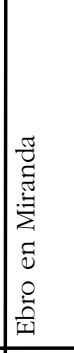 & 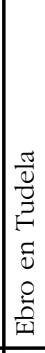 & 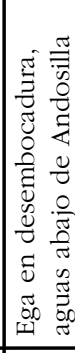 & 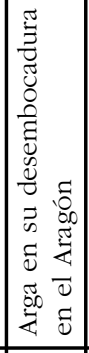 & 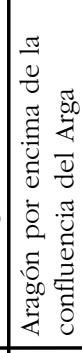 & 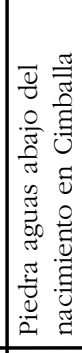 & 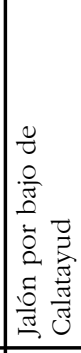 & 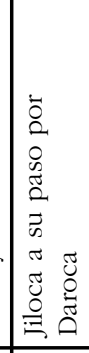 & 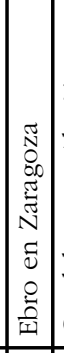 & 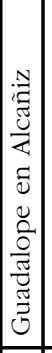 & 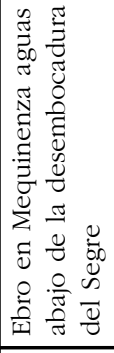 \\
\hline & 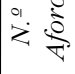 & $\hat{\mathrm{\lambda}}$ & $\infty$ & in & in & in & $\infty$ & $\begin{array}{l}\infty \\
\infty\end{array}$ & $\infty$ & & & $\stackrel{\infty}{\underset{I}{J}}$ \\
\hline
\end{tabular}




\begin{tabular}{|c|c|c|c|c|c|c|c|c|c|c|c|c|}
\hline & జ్๊ & 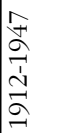 & 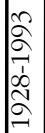 & 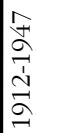 & 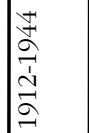 & 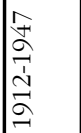 & 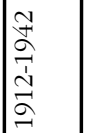 & 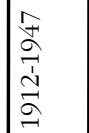 & 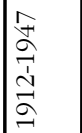 & 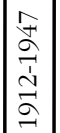 & 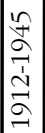 & 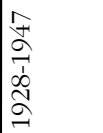 \\
\hline & 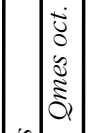 & 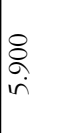 & $\mid$\begin{tabular}{l}
0 \\
0 \\
0 \\
0 \\
0 \\
\hdashline \\
0
\end{tabular} & $\underset{-}{\stackrel{\leftrightarrow}{\circ}}$ & $\begin{array}{l}8 \\
\text { o. } \\
\text { in }\end{array}$ & 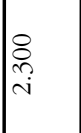 & 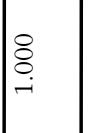 & 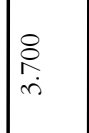 & $\mid$\begin{tabular}{l}
8 \\
\hdashline \\
0
\end{tabular} & 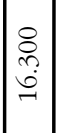 & $\mid$\begin{tabular}{l}
0 \\
\multirow{1}{0}{} \\
0
\end{tabular} & $\begin{array}{l}8 \\
\vdots \\
0 \\
0 \\
0\end{array}$ \\
\hline & 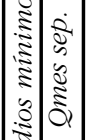 & $\begin{array}{l}8 \\
8 \\
\infty \\
+ \\
+\end{array}$ & $\mid \begin{array}{l}0 \\
10 \\
0 \infty \\
i n\end{array}$ & @ & 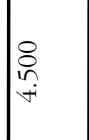 & $\begin{array}{l}8 \\
0 \\
0 \\
0\end{array}$ & $\mid \begin{array}{l}8 \\
\infty \\
0 \\
0\end{array}$ & $\underset{-}{\searrow}$ & 离 & $\left|\begin{array}{c}8 \\
\vdots \\
\hdashline\end{array}\right|$ & $\left|\begin{array}{l}8 \\
1 \\
0 \\
0\end{array}\right|$ & $\begin{array}{l}8 \\
\vdots \\
\infty \\
\infty\end{array}$ \\
\hline & 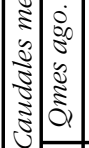 & $\begin{array}{l}8 \\
\text { in } \\
\text { in }\end{array}$ & $\mid \begin{array}{l}8 \\
0 \\
0 \\
\text { in } \\
\text { in }\end{array}$ & ì & ஓ̊̀. & \&. & $\mid \begin{array}{l}8 \\
\infty \\
\infty \\
0 \\
0\end{array}$ & 怘 & 亩 & 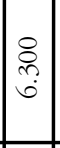 & $\mid \begin{array}{c}0 \\
0 \\
0 \\
0\end{array}$ & $\begin{array}{l}\vdots \\
\vdots \\
\vdots \\
m\end{array}$ \\
\hline & 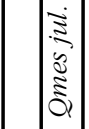 & ס్రి & $\mid \begin{array}{l}2 \\
\vdots \\
\infty \\
\infty \\
1\end{array}$ & : & ஓ্̀. & $\begin{array}{l}\dot{0} \\
\dot{0} \\
\dot{m}\end{array}$ & 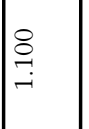 & 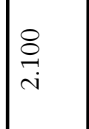 & $\stackrel{8}{\stackrel{1}{0}}$ & $\left|\begin{array}{l}8 \\
8 \\
\hdashline \\
\hdashline\end{array}\right|$ & $\mid \begin{array}{l}8 \\
0 \\
1 \\
0 \\
0\end{array}$ & 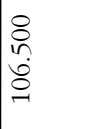 \\
\hline 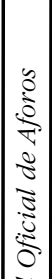 & 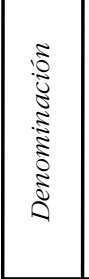 & 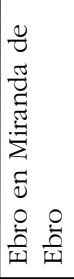 & 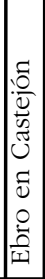 & 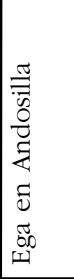 & 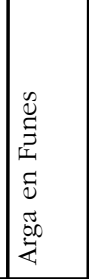 & 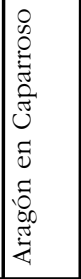 & 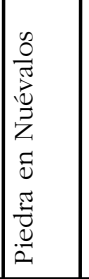 & 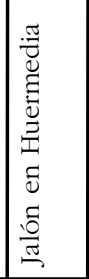 & 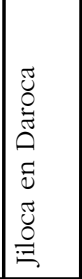 & 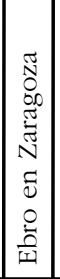 & 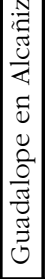 & 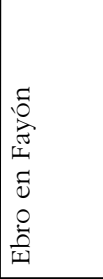 \\
\hline$\approx$ & 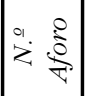 & - & $\mathrm{N}$ & n & $\psi$ & in & $\infty$ & a & 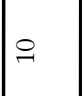 & $=$ & $\approx$ & 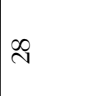 \\
\hline & \begin{tabular}{l}
$\bar{\Xi}$ \\
\multirow{3}{3}{} \\
0
\end{tabular} & & 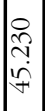 & 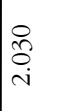 & 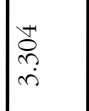 & 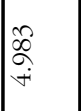 & 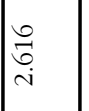 & 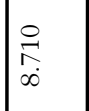 & $\begin{array}{l}\stackrel{2}{2} \\
\hat{n} \\
\hat{n}\end{array}$ & $\mid$\begin{tabular}{c|}
$i n$ \\
$\vec{a}$ \\
$\infty$ \\
$\dot{v}$
\end{tabular} & 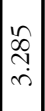 & 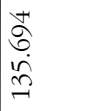 \\
\hline 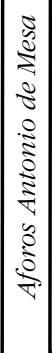 & 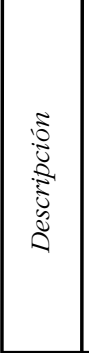 & 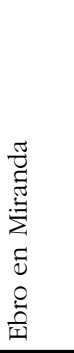 & 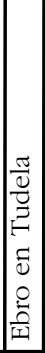 & 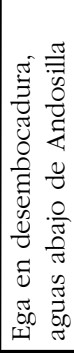 & 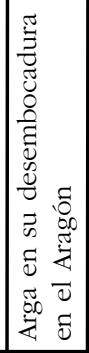 & 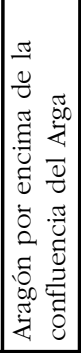 & 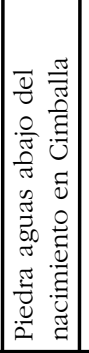 & 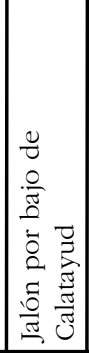 & 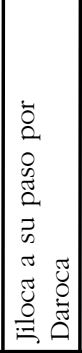 & 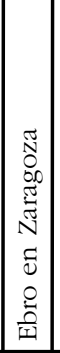 & 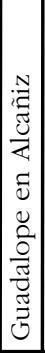 & 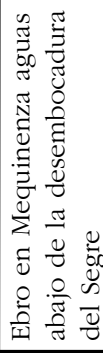 \\
\hline & $\left|\begin{array}{ll}0 & 0 \\
z & 5 \\
\hdashline\end{array}\right|$ & ๙ે & $\infty$ & in & $\stackrel{\infty}{n}$ & in & $\infty$ & $\mid \infty$ & $\infty$ & $\bar{\sigma}$ & Ĩ & $\stackrel{\infty}{\underset{I}{J}}$ \\
\hline
\end{tabular}


los caudales de estiaje en Zaragoza y Tortosa son de 28 y $136 \mathrm{~m}^{3} / \mathrm{s}$, y que si alguna desconfianza merecen "proviene de haberse hecho estando ya la estación muy adelantada y ser dudoso que el río estuviera en estiage (sic); y porque hemos tenido ocasión de observar y oír desde Peña-Labra a los Alfagues y desde la Cerdaña al Moncayo, que el estiage (sic) que hemos estudiado no puede reputarse como de los más estériles que se conocen en este río" (op.cit). Valores que hemos visto resultan totalmente razonables (véase tabla 1 y 2) y que curiosamente, quien sabe si por mera coincidencia, se encuentran en el mismo orden de magnitud de los caudales mínimos que actualmente el Organismo de Cuenca fija para ambos puntos, $30 \mathrm{~m}^{3} / \mathrm{s}$ en Zaragoza y 100 $\mathrm{m}^{3} / \mathrm{s}$ en desembocadura.

\section{La agricultura y el regadío en el valle del Ebro}

Al margen de los trabajos de aforo, uno de los aspectos de mayor interés es que a lo largo del documento, Antonio de Mesa da numerosas noticias sobre el estado de la agricultura en la cuenca del Ebro, realizando una primera evaluación del regadío. En las páginas descriptivas iniciales extrae a partir de los datos de los Anuarios de Estadística de 1858 y 1860 las hectáreas, según el Registro de la Propiedad, cultivadas por provincias en el valle del Ebro, obteniendo unas cifras de 225.674 ha de riego y 3.217.391 de secano, si bien Antonio de Mesa dirá "cuando me ocupe de los aprovechamientos de agua de este valle, tendré ocasión de observar que estos números son inadmisibles" (op.cit). También recoge los tipos de cultivo cifrando en un $75 \%$ las tierras regables que se dedicarían a cereal, mientras que el resto se lo reparten casi equitativamente entre los viñedos, olivares y prados.

De la Región Superior o cabecera del Ebro hasta Miranda dirá que "los riegos no tienen aplicación ninguna en la región superior, donde no existen en la actualidad, si se esceptúan (sic) las pocas huertas..., cuya estensión total no creemos llegue a 500 hectáreas" (op.cit). Para la Región Media, desde Miranda a Zaragoza estima que los riegos "sólo se elevan á 65000 hectáreas" (op.cit), una tercera parte en el eje del Ebro, principalmente regadas por los canales Imperial y Tauste, mientras que para la Inferior, desde Zaragoza a desembocadura y que comprende los riegos del Canal de Urgel y Cherta, alcanza las 93.000 hectáreas. Haciendo un total de 158.516 hectáreas de riego según el texto -o 159.516 según la suma de los valores parciales-, que en las conclusiones del informe se transcriben erróneamente por 185.516 (tabla 4). Basa sus apreciaciones "en el agua que se consume y en apreciaciones hechas a la vista" (op.cit). En 1904 la cifra teórica de regadío era de 389.539 ha, con sólo el 50\% de cereales, que seguramente debe ser corregida por la más realista que establece Lorenzo Pardo para 1924 de 406.134 ha, y de ellas 285.000 de riego continuo y regular, (Omedas Margelí et al. 1994, citando a Eugenio Nadal; Lorenzo Pardo, 1931), por 
Tabla 3. Listado de aforos efectuados por Antonio de Mesa en el Reconocimiento Hidrológico del Valle del Ebro.

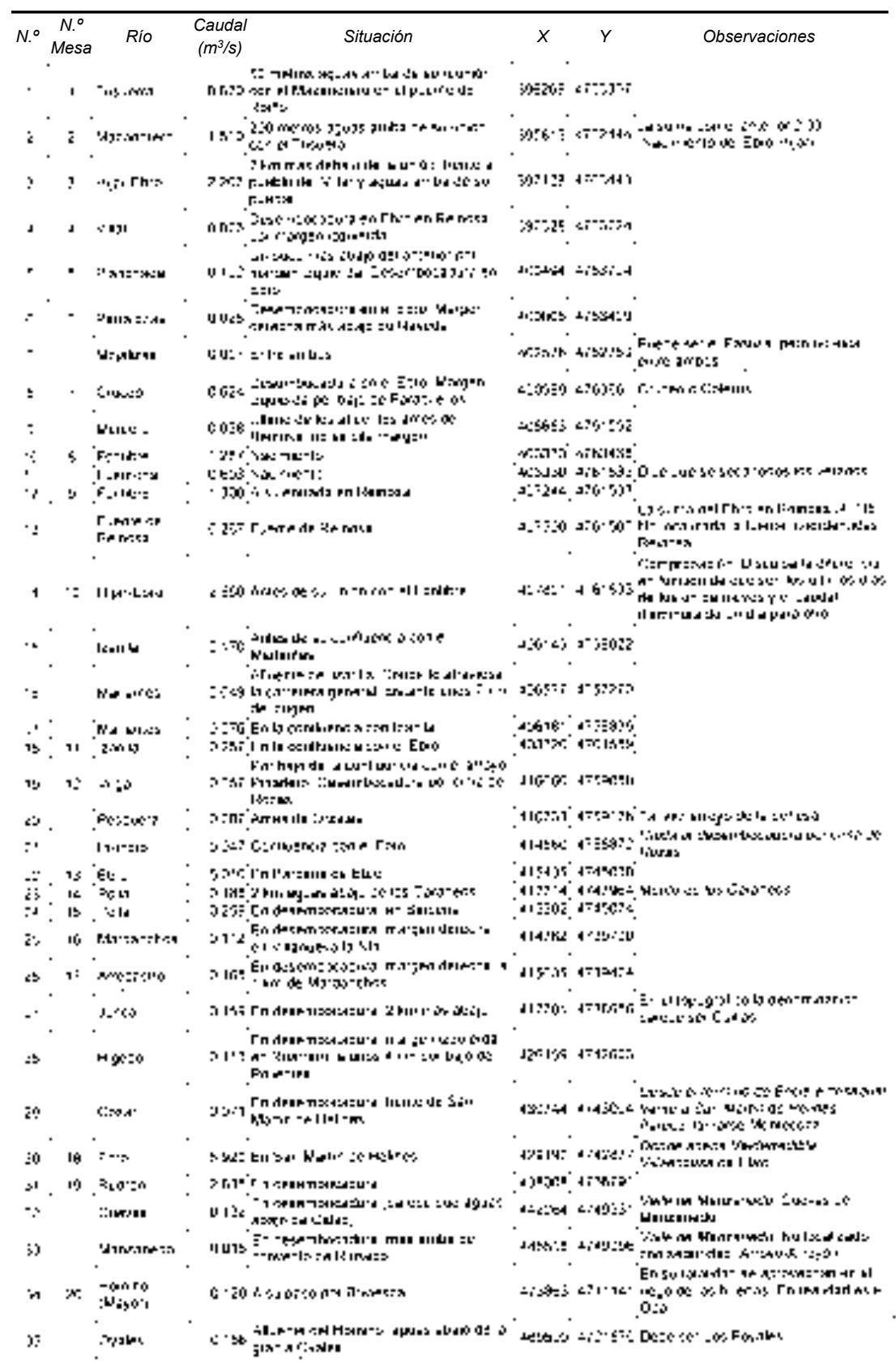




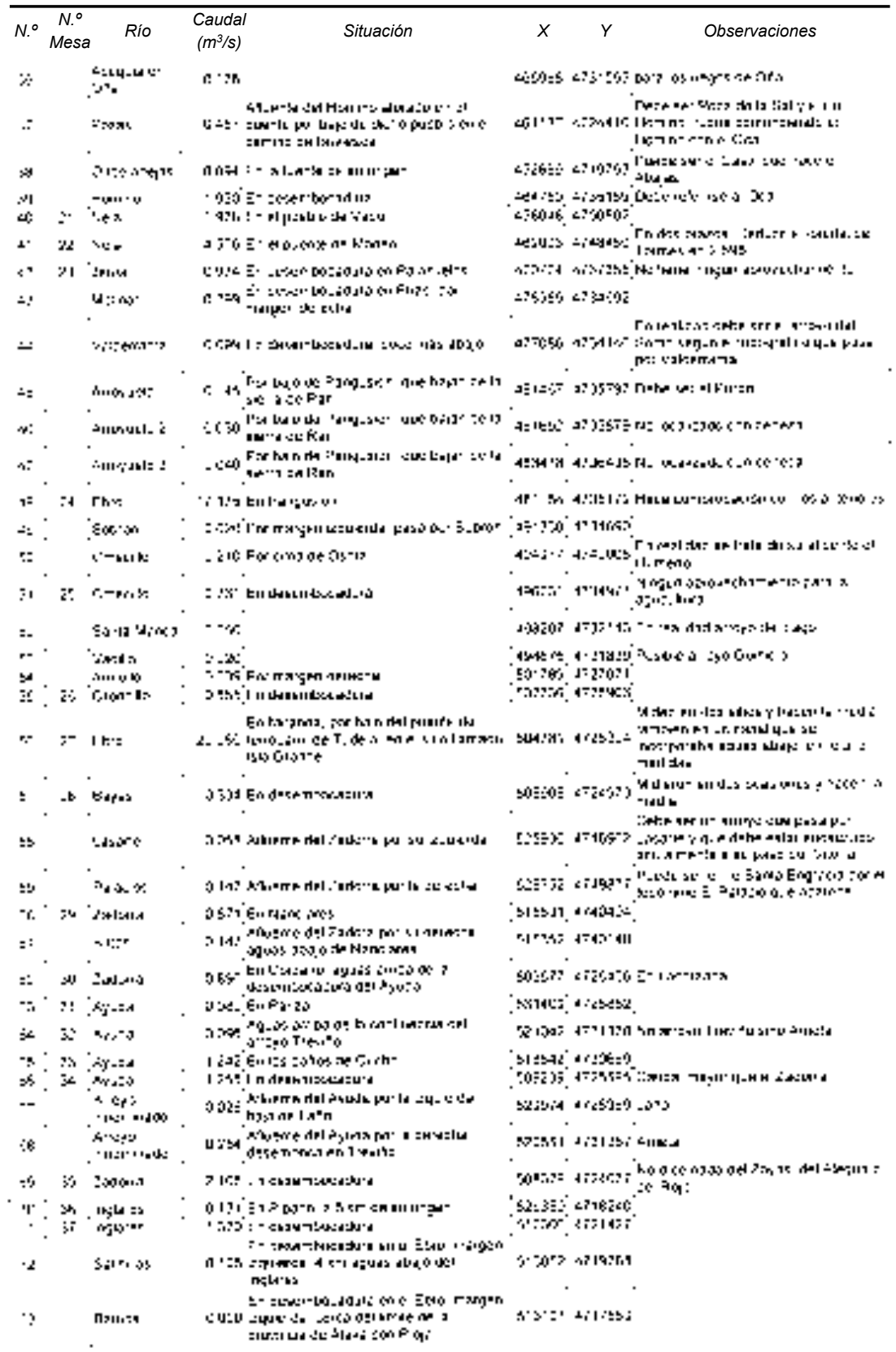




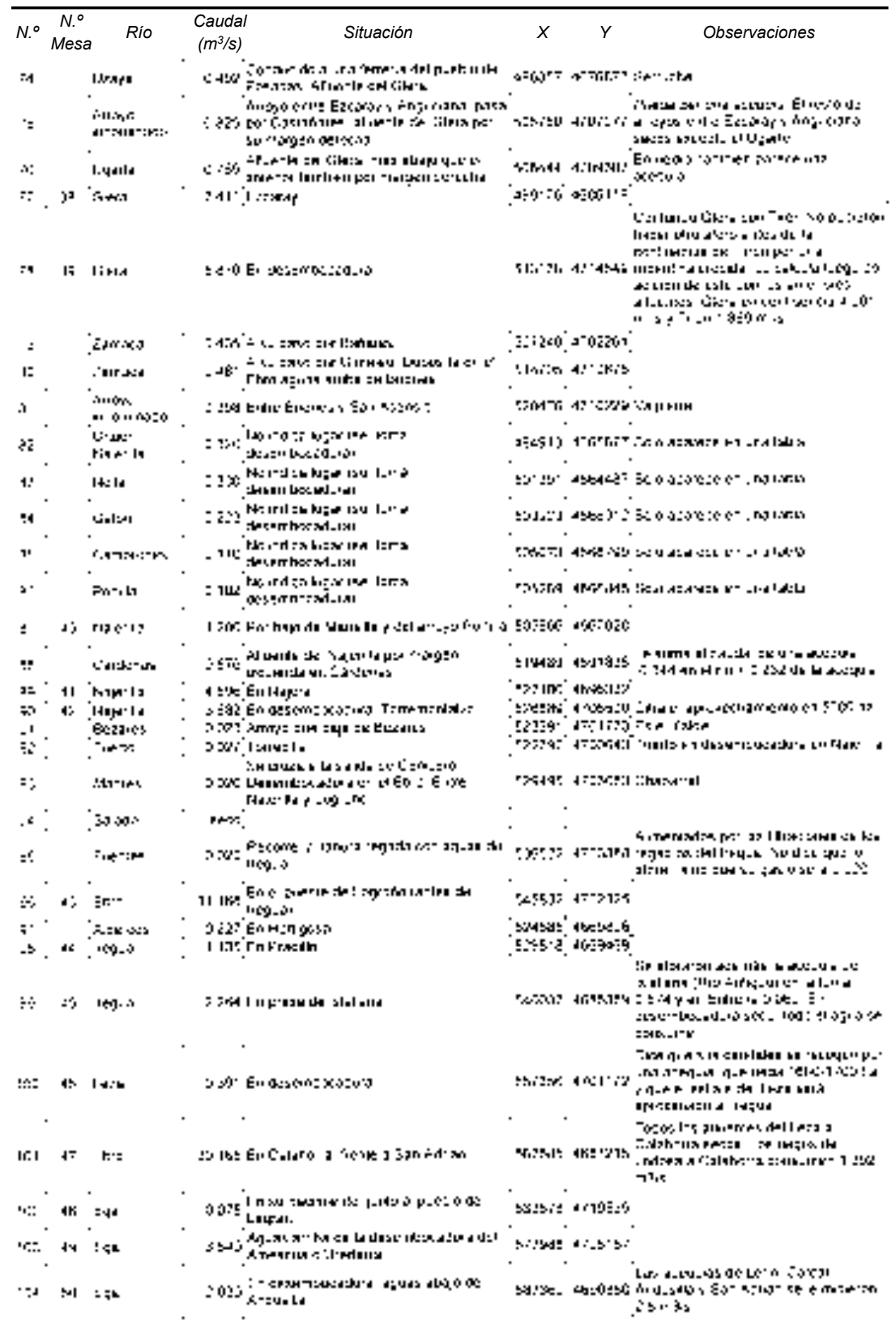




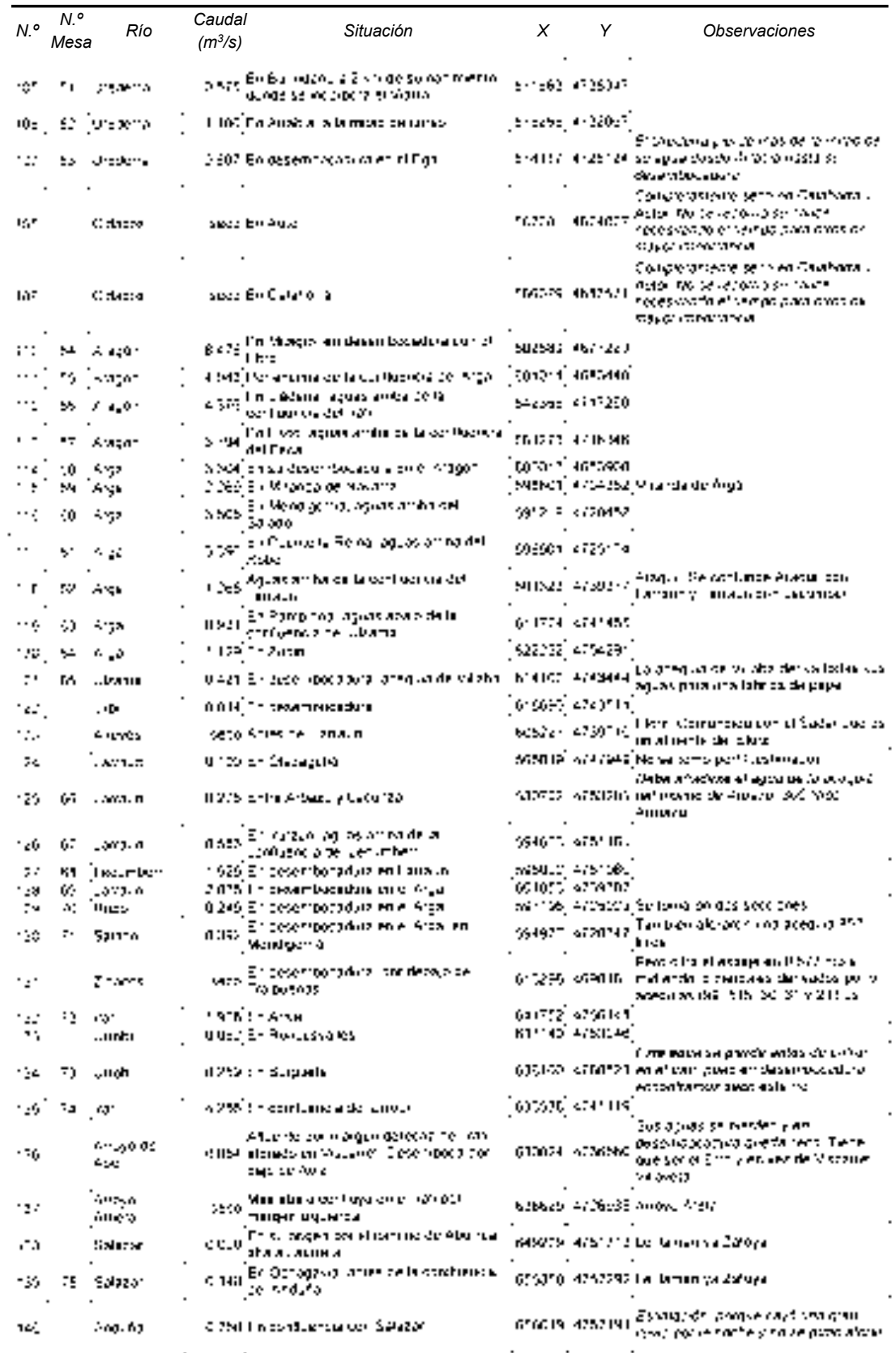




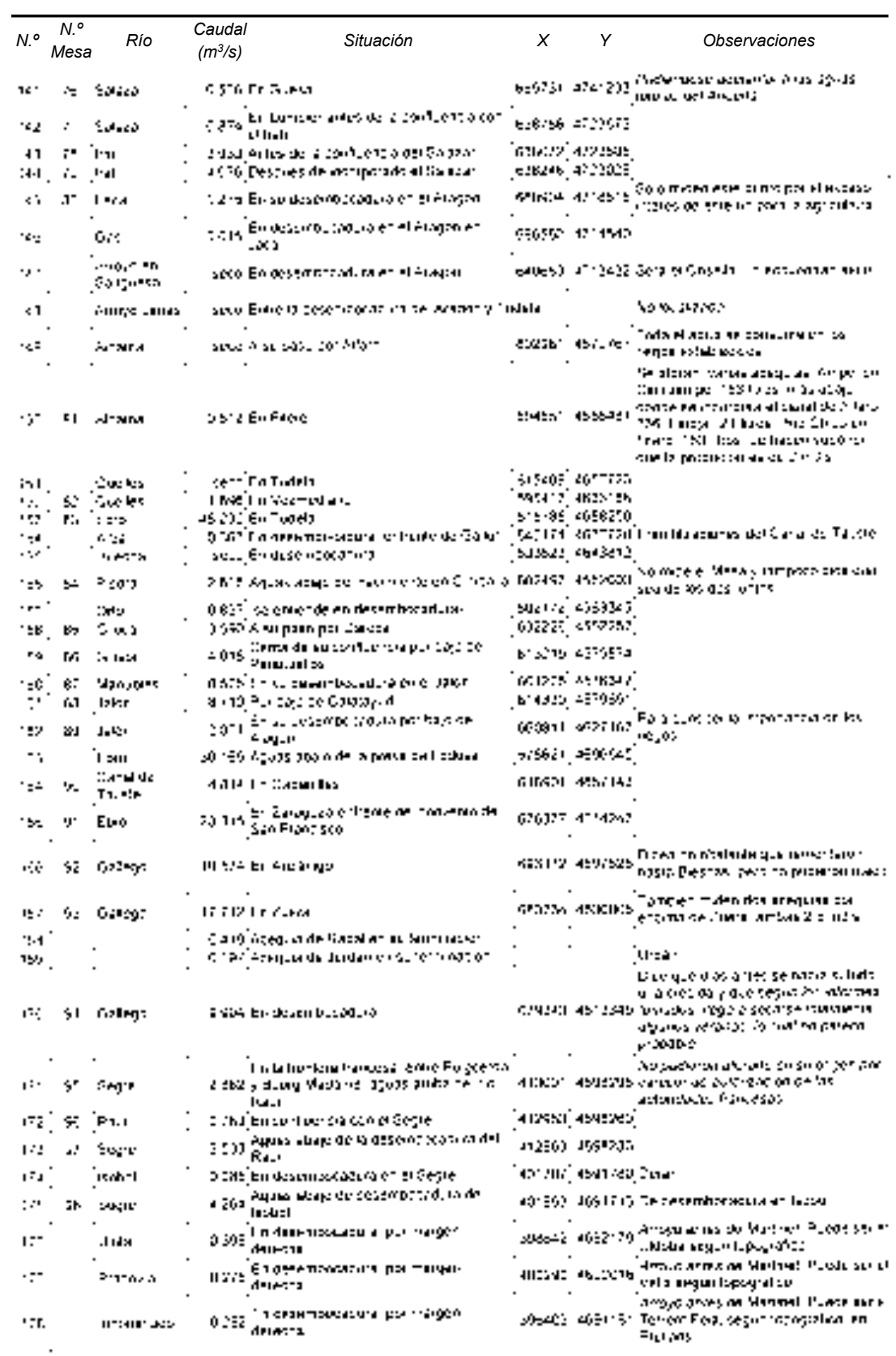




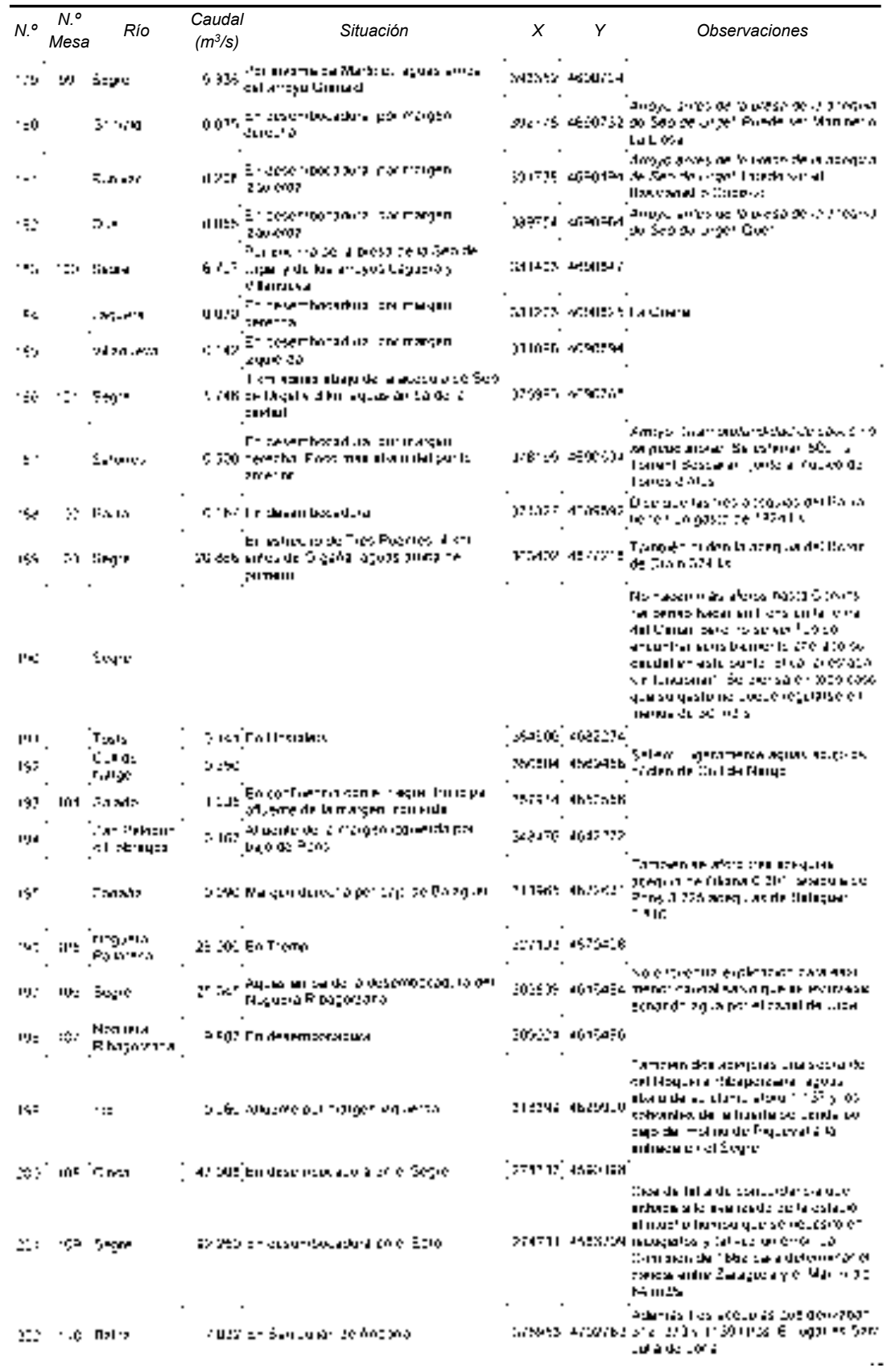




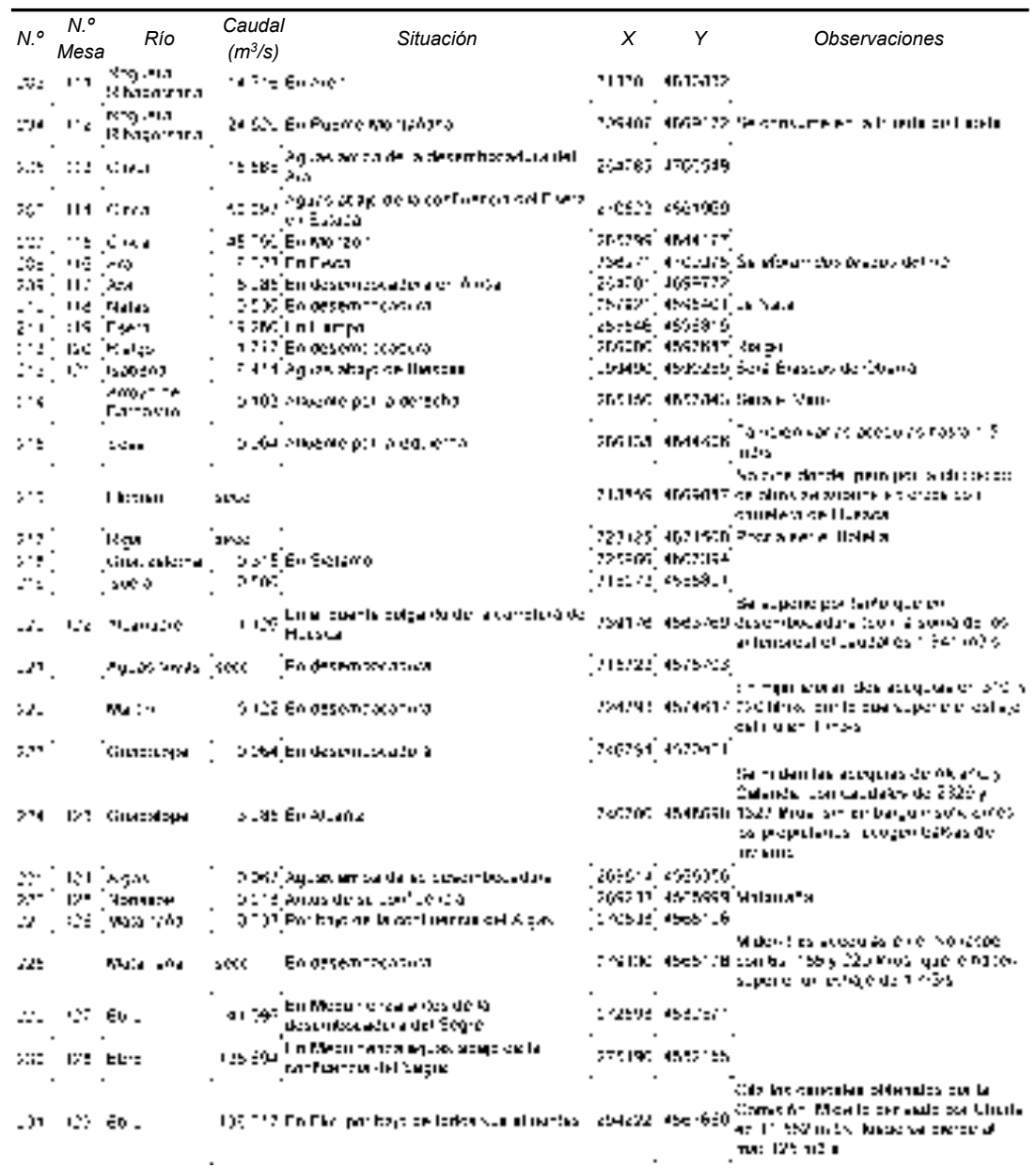

Fuente: Obra citada y elaboración propia.

lo que no cabe duda que, a pesar de las dificultades, el esfuerzo realizado durante la Restauración por aumentar los regadíos tuvo frutos sustanciales en la cuenca del Ebro.

Ya hemos indicado cómo los riegos consumían el agua del estiaje en varios ríos de la margen derecha sin dar satisfacción a todas las necesidades. Para mejorar la situación de los riegos en estas zonas propone acumular el agua invernal ampliando el número de balsas que en algunos lugares los particulares ya venían ejecutando como en el caso de Tudela que, sometida a grandes escaseces "por el despilfarro que 
se hace (de las aguas del Queiles) en su parte superior... ... se ven obligados a proporcionarse las más indispensables por medio de balsas o estancas... ... Lástima es que ya que se tuvo esta feliz idea no se desarrolle a mayor escala" (Antonio de Mesa, 1865). También refiere lo mismo para el caso del Guadalope donde los propietarios se ven obligados "a recoger en balsas las (aguas) sobrantes de invierno, y a pensar en nuevos proyectos de pantanos y otros artificios para ocurrir a esta necesidad' (op.cit).

En las conclusiones finales, refiere además las empresas de riego que "bajo el punto de vista del interés general podrían promoverse en este valle" (Antonio de Mesa, 1865), en realidad proyectos que ya estaban en la mente de algunos visionarios, o incluso contaban con proyecto o concesión. Lo resume en seis canales, de ellos tres en el propio eje del Ebro, básicamente coincidentes con el actual Canal de Lodosa, otro desde el Aragón para regar las vertientes del Arba, el que en el futuro sería Canal de Bardenas, un quinto de Tamarite desde el Cinca y Ésera para la parte baja de la provincia de Huesca, embrión de lo que hoy es el Canal de Aragón y Cataluña, y un sexto desde el Segre o el Ebro para regar los campos de Tarragona, que en cierto modo sería asimilable al Canal de la Margen Izquierda del Delta. No cita el proyecto del Canal del Sobrarbe, antecedente de Riegos del Alto Aragón, bien es cierto que se trata de un proyecto de 1865, aunque en las páginas interiores citaría una concesión para estudiar un canal que partiendo del Cinca encontraría otro del Gállego.

Finalmente, es testigo de la tremenda transformación y de la "riqueza colosal" gracias al canal de Cherta, que estaba significando el cultivo desde 1858 de arroz en el Delta y cuyas tierras "cuyo valor apenas se elevaba hace pocos años a 200 reales por hectárea, hoy se paga a 4000 reales, y no se encuentra quien venda, con la esperanza de que ha de valer más"(op.cit).

\section{Otros elementos de interés}

La descripción del medio físico es concisa, la del paisaje humano casi anecdótica, aunque recoge descripciones de actividades relacionadas con el agua hoy perdidas, como cuando cita la importancia de la navegación en el Ebro donde la ciudad de Tortosa "tiene 45 barcos matriculados y hace un tráfico de 20.000 toneladas" (op.cit), o por otro lado, la manera de ejecutar las operaciones de transporte de la madera por el Irati, supliendo el insuficiente caudal mediante diques de retención, a manera de esclusas, o también, el procedimiento para la extracción de la sal en Poza de la Sal.

Descarta ya por descabellada "la manía favorita en cierta época de hacer navegables nuestros ríos" (op.cit) con grandes canales de navegación que a lo largo y ancho 
Tabla 4. Superficie de regadío por subcuencas calculada por Antonio de Mesa en el Reconocimiento hidrológico del valle del Ebro.

\begin{tabular}{|l|c|}
\hline \multicolumn{1}{|c|}{ Río } & $\begin{array}{c}\text { Superficie } \\
\text { ( } \mathrm{ba} \text { ) }\end{array}$ \\
\hline Cabecera del Ebro & 500 \\
\hline Zadorra & 100 \\
\hline Glera y Tirón & 3.000 \\
\hline Zamaca & 2.000 \\
\hline Najerilla & 2.000 \\
\hline Iregua & 3.000 \\
\hline Leza & 2.500 \\
\hline Ega & 3.000 \\
\hline Cidacos & 3.000 \\
\hline Aragón, Arga y Zidacos & 6.000 \\
\hline Alhama & 3.000 \\
\hline Queiles & 5.000 \\
\hline Jalón & 8.000 \\
\hline Ebro. Lodosa y Calahorra & 2.000 \\
\hline Ebro. Tudela & 2.000 \\
\hline Ebro. Canal de Tauste & 6.589 \\
\hline Ebro. Canal Imperial & 13.827 \\
\hline Gállego & 8.000 \\
\hline Huerva & 1.000 \\
\hline Aguas Vivas & 500 \\
\hline Martín & 1.500 \\
\hline Guadalope & 5.000 \\
\hline Matarraña & 1.000 \\
\hline Segre & 60.000 \\
\hline Canal de Cherta. Delta del Ebro & 17.000 \\
\hline Total & 159.516 \\
\hline
\end{tabular}

Fuente: Obra citada.

de la cuenca se habían pensado, no sólo los del propio Ebro sino los que se proponían para unir el valle del Duero con el Ebro, o este último con el Cantábrico.

En el reconocimiento se enumeran y describen concisamente algunas de las lagunas, manantiales o explotaciones de sal. Las lagunas que cita son las de Añavieja en el Alhama, provincia de Soria, que más adelante sería desecada "con una estensión (sic) de 1000 hectáreas" (op.cit) y que es aprovechada para los riegos de Ágreda y Cervera de Río Alhama, Herbia (Hervías) en La Rioja, en la divisoria del Glera y Nájera, actualmente en proceso de restauración, y que es de tal pequeña entidad que sorprende su cita si no fuera porque pasaran por su lado, y la de Urbión, en la cabecera del Najerilla, permanente de 2 hectáreas "notable por su grande elevación....... su profundidad muy grande, según los naturales del país, cuyas tentativas para ave- 
riguarla han sido inútiles" (op.cit). Curiosamente, de la laguna de Sariñena, en la provincia de Huesca, dirá que es de muy poca importancia. También hace referencia a los terrenos pantanosos del Delta del Ebro y se extiende algo más en la laguna de Gallocanta, en la provincia de Zaragoza, cifrando su extensión en 1800 hectáreas, haciendo referencia a las fiebres que originaba, "las intermitentes hacen frecuentes estragos", y a los proyectos para su desagüe de los que "no puede resolverse la cuestión de utilidad como negocio, si bien bajo el punto de vista de la salubridad de este país, no parece ofrecer duda" (op.cit).

Las fuentes de aguas minerales y medicinales que menciona son: Fuente de la Salud en Sobrón (Álava), Corconte (Cantabria), Baños de Cucho en el Ayuda (Álava) Baños de Panticosa (Huesca), Salinillas cerca de las Conchas de Haro (La Rioja), Tiermas en el Aragón (Huesca), Arive en el Irati (Navarra), Arnedo y Arnedillo en el Cidacos (La Rioja), Fitero, Cervera y Grávalos en el Alhama (Navarra y La Rioja) y Alhama de Aragón (Zaragoza).

En cuanto a las salinas: Poza de la Sal en el Homino, Añana en el Omecillo, Rosío en el Nela, Herrera y Buradón en la provincia de Burgos, Remolinos y Sástago en el Ebro, Ojos Negros en el Jiloca, Armillos y Valtablado en el Martín, Naval en el Cinca, Peralta en el Sosa, Gerri en el Noguera-Pallaresa, Villanueva en el Segre y Alfaques (Alfagues) en el Delta.

\section{Conclusiones}

En definitiva, el Reconocimiento hidrológico del Valle del Ebro efectuado por la brigada hidrológica dirigida por Pedro Antonio de Mesa en 1863 y el informe subsiguiente realizado por este mismo Ingeniero en 1865, es digno de ser recordado no sólo por su carácter pionero, por la labor encomiable de su impulsor y realizador, por el esmero en su elaboración o por el hito que representa en la Historia de la Geografía en España, sino también porque adquiere para el momento presente un extraordinario valor documental, como testimonio de la evolución operada en la cuenca.

Este valor documental se manifiesta en varios aspectos que a lo largo de este trabajo se han citado. Entre ellos quizá puedan destacarse dos: la foto fija de las superficies de regadío de la cuenca del Ebro en 1863 y la afección ya en aquella época del régimen hidrológico veraniego, con varios ríos como el Iregua, el Queiles, el Cidacos, el Alhama, y seguramente otros, si atendemos a que el estiaje de 1863 "no puede reputarse como de los más estériles que se conocen", que quedaban secos en sus desem- 
bocaduras en verano, ¿tal vez el Gállego?, y que sólo las regulaciones posteriores han corregido aunque al precio de alterar el régimen invernal, en ocasiones invirtiendo ambos. Interesante circunstancia que nos muestra cómo la alteración de los ecosistemas hídricos de muchos tramos bajos de ríos de la cuenca del Ebro se viene produciendo desde hace siglos.

\section{Bibliografía}

Antonio de Mesa, Pedro (1864): Reconocimiento bidrológico del valle del Guadalquivir. Junta General de Estadística. Madrid.

Antonio de Mesa, Pedro (1865): Reconocimiento hidrológico del valle del Ebro. Junta General de Estadística. Madrid.

Bolea Foradada, Juan Antonio (1986). Los riegos de Aragón. Grupo Parlamentario Aragonés Regionalista. Huesca.

Lorenzo Pardo, M. (1916). Proyecto del Pantano del Ebro. Archivo de la Confederación Hidrográfica del Ebro. Zaragoza.

Lorenzo Pardo, M (1931). La conquista del
Ebro, Confederación Hidrográfica del Ebro. Zaragoza.

Mateu Bellés, J. F.(1996): Las brigadas hidrológicas de la Junta General de Estadística (1859-1867). Saitabi, Volum Extraordinari, 251-281, Valencia.

Muro Morales, J. I., Nadal Piqué, F., Urteaga González, L. (1995). Els estudis hidrologics de Pedro Antonio de Mesa (1862-1865). Treballs de la Societat Catalana de Geografia, nº 40, vol X, 33-41

Omedas Margelí, M., Brotons Florián, J. (1994) La evolución de los regadios en la cuenca del Ebro. Confederación Hidrográfica del Ebro. Informe inédito. 